\title{
Progresses in Gene Therapy of Neurodegenerative Disorders
}

\author{
Ashim Kumar Basak and Tridip Chatterjee* \\ Department of Molecular Biology, Institute of Genetic Engineering, India
}

Received: 此 January 25, 2018; Published: 跳 February 15, 2018

*Corresponding author: Tridip Chatterjee, Department of Molecular Biology, Institute of Genetic Engineering, India

\begin{abstract}
Gene therapy is a technique that uses genes to treat or prevent diseases. Gene therapy to the brain faces other difficulties in addition to the general issues one faces with gene therapy. A major limiting factor for the delivery of gene to the brain is the blood brain barrier (BBB), which most gene expression vectors can not cross naturally. However, recent advances in gene transfer technology have generated promises for delivery of therapeutic genes to brain. In this small review we shall make a discussion on the recent developments of gene therapies for some important neurodegenerative disorders.
\end{abstract}

Keywords: Gene therapy; Viral vectors; Alzheimer's disease; Parkinson's disease; Huntington disease; Canavan disease; X-Linked adrenoleukodystrophy.

\section{Introduction}

Gene therapy is the administration of genetic materials into the somatic cells of a patient that exert therapeutic effects by correcting genetic defects, over expression of desired proteins or inhibition of the expression of unwanted harmful proteins [1]. For primary neurodegenerative disorders patient's condition progressively deteriorated and the current therapies remain limited to symptomatic treatments [2]. The presence of blood-brain barrier (BBB) prevents the delivery of therapeutic agents to the brain [3]. However, recent advances in gene transfer technologies show promise for delivery of therapeutic genes to brain for the replacement of defective proteins, neuroprotection, restoration of neuronal functions etc [2]. With the advent in the understanding the molecular pathologies, it is anticipated that the therapeutic genes have the ability of revolutionize the management to human diseases [4].

\section{Strategies for gene therapy in neurodegenerative disorders}

There may be two categories of gene therapy for neurodegenerative disorders namely- in vivo and ex vivo gene therapies. In the ex vivo approach, the therapeutic gene is introduced into the neuronal and non-neuronal cell by vectors and later is transferred into the appropriate brain regions. The graft can synthesize neurotransmitters for specific pathologies such as dopamine (DA) for Parkinson's disease (PD), acetylchilone for Alzheimer's disease (AD) or GABA for Huntington disease [5]. The in vivo gene therapy in neurodegenerative disorders involves direct infusion of genes in specific brain regions through neurosurgical stereotactic injection [3]. Vectors for gene delivery in brain for neurodegenerative disorders may be of two types- synthetic and viral vectors. Synthetic vectors such as cationic lipids and cationic polymers can complex with the nucleic acid by electrostatic force of attraction and can be associated with specific ligand for cellular or nuclear targeting for delivering genes in desired sites in the brain [5].Viral vectors are designed in such a way that, some parts of their genome are removed and replaced by desired genes for delivery into the host cells. Such vectors are available at present from retrovirus, adenovirus, adeno-associated virus (AAV), herpes simplex virus (HSV) and lentivirus. All the viral vector systems have some limitations. However, strategies have been developed to overcome the toxicities associated with the uses of viral vectors $[2,6]$. A number of clinical trials in operation now are using viral and non-viral vectors gene therapy in neurodegenerative disorders such as AD, PD, Batten disease, Canavan disease, X-Linked Adrenoleukodystrophy (X-ALD) etc. Many of them are in phase I, phase II and even in phase III stages of clinical trials and have 
shown promises in the effectiveness of the therapies [3].

\section{Recent progresses of gene therapy for some neurodegenerative disorders}

Alzheimer's disease (AD) is the most common neurodegenerative disorder associated with the progressive damages in the brain regions important for cognitive functions such as neocortex, hippocampus, amygdale etc. and cause serious damages in the cholinergic neurocircuits [5]. The hallmark of pathology of $\mathrm{AD}$ includes the formation of extracellular amyloid plaques and intracellular neurofibrillary tangles (NFT) that cause the progressive loss of neurons and synapses [7]. There are two forms of this disease namely- familial early-onset AD (EOAD) and sporadic late-onset $\mathrm{AD}$ (LOAD) of which the former includes upto $6 \%$ of all patients in the age of 30-60 years. In the later case the age of onset is usually after 65 years [8]. The familial EOAD arises due to over production of a protein called amyloid beta $(A \beta)$ due to the mutations in three genes namely- amyloid precursor protein (APP), presenilin1 (PSEN1) and presenilin 2 (PSEN 2). In contrast LOAD results from a combination of genetic and environmental factors. The major genetic risk factor is the $\mathrm{E} 4$ allele of the apolipoproten E (APOE) [9,10]. Since nerve growth factor (NGF) prevents degeneration of adult cholinergic neurons in the fore brain after injury [11], primary fibroblast, neural stem cells, progenitor cells were genetically modified by transferring NGF genes retrovirally and were transplanted in the brain of experimental rodents. It was observed that cell grafts prevented spontaneous age related cholinergic atrophy and reversed their cognitive impairments in these animals $[12,13]$. Clinical trials of gene therapy for $\mathrm{AD}$ are based on intracerebral delivery of AAV that encodes NGF. One clinical trial revealed that NGF infusion into basal forebrain bilaterally exhibited safety and tolerability of the procedure in patients for at least two years after injection that lowered cognitive decline and showed persistent bioactivity in the autopsy [3]. In another study, among 10 patients, degenerating neurons in the $\mathrm{AD}$ brain responded to NGF. All patients exhibited a trophic response to NGF in the form of axonal sprouting toward the NGF source. In 3 patients who underwent unilateral gene transfer, cholinergic neuronal hypertrophy occurred on the NGF-treated side of the brain. Activation of cellular signaling and functional markers were present in 2 patients who underwent adeno-associated viral vectors -mediated NGF gene transfer [14].

Parkinson's disease (PD) is a neurodegenerative disorder characterized by the loss of dopaminergic neurons of substantia nigra and exhibits clinical symptoms like tremor, rigidity, postural instability etc. Gene therapy of PD relies on two strategies i.e. i) resoration of dopamine (DA) level in brain by the therapeutic delivery of gene encoding human tyroxine hydroxylase $(\mathrm{TH})$, a rate limiting enzyme in DA synthesis and ii) neuroprotective measure to prevent loss of dopaminergic neurons of substantia nigra by delivering the neurotrophic factors genes [5]. Direct infusion of defective HSV carrying the TH gene into the striatum of animal model of PD had reduced motor dysfunctions of PD. Biochemical recovery included increases in both striatal tyrosine hydroxylase enzyme activity and in extracellular dopamine concentrations [15]. Loss of DA neurons of substantia nigra is also linked to the dysregulation of inhibitory and excitatory neurotransmission. Inhibitory neurotransmitter GABA synthesis is catalyzed by the enzyme glutamatic acid decarboxylase (GAD). Clinical trials that involved direct infusion of AAV vectors encoding GAD into the subthalamic nucleus exhibited the improvement of the motor symptoms of PD mainly on the side of the body contralateral to the treatment site in the brain without any adverse effect [16].

Huntington disease (HD) is a neurodegenerative disorder characterized by chorea, dystonia, cognitive deficiencies etc. resulting from the mutation of Huntingtin gene (HTT) that overcausing the polyglutamine expansion in the huntingtin proteinthat encodes glutamine. Overexpansion of the trinucleotide renders the huntingtin protein to attain an abnormal conformation with cytotoxic effect [17]. Silencing of HTT gene through RNA interference conducted by siRNA delivery by AAV in the brain has shown efficacy in mouse model of HD [18]. The first clinical trial of gene therapy of HD is in progress. It uses antisense oligonucleotides that do not distinguish between mutant and normal HTT mRNA. It is anticipated that suitable vector mediated knock out of mutant HTT and its replacement with wild copy of HTT gene will provide next generation of therapies for HD [17]. Canavan disease is a rare autosomal recessive leukodystrophy caused by the mutation of ASPA gene encoding aspartate cyclase. Due to the mutation of this gene and loss of enzymatic activity of the protein thereby, increased concentration of its substrate i.e. $\mathrm{N}$-acetyl aspartate (NAA) is accumulated in the brain leading to spongiform degeneration of white matter and severe impairment of psychomotor development [3]. It has been reported that intraparenchymal delivery of AAV vector carrying ASPA gene in a number of brain sites of many Canavan disease patients resulted in the decrease of the elevation of NAA, slowing down of brain atrophy and improvement of seizure frequency associated with this disease [19]. X-linked Adrenoleukodystrophy (X-ALD) is a neurodegenerative disease in which demeyelination occurs due to the mutation of ABCD1 gene and loss of function of ALD protein. Gene therapy for this disease requires the patients to be in the early stage of the disease. In one study infusion of autologous CD34+ cells in 17 boys with a lentiviral vector that contained ABCD1 cDNA resulted into functional expression of ALD protein. Results of this study suggested the promise of gene therapy in early-stage cerebral adrenoleukodystrophy [20].

\section{Discussion}

The use of gene therapy to target the central nervous system presents many technical and biological challenges. These may be overcome by using proper gene vector delivery strategies. Overall 
recent experimental and human studies suggest that gene therapy for neurodegenerative disorders exhibits good prospect.

\section{References}

1. Rubanyi GM (2010) Gene Therapy-Basic Principles and the Road from Bench to Bedside. Pharmaceutical Sciences Encyclopedia 30: 1-28.

2. Carter JE, Schuchman EH (2001) Gene therapy for neurodegenerative diseases: fact or fiction? Br J Psychiatry 178: 392-394.

3. Françoise Piguet, Sandro A, Nathalie C (2017) Clinical Gene Therapy for Neurodegenerative Diseases: Past, Present, and Future. Human Gene Therapy. Hum Gene Ther 28(11): 988-1003.

4. Lee CS, Bishop ES, Zhang R, Yu X, Farina EM, et al. (2017) AdenovirusMediated Gene Delivery: Potential Applications for Gene and Cell-Based Therapies in the New Era of Personalized Medicine. Genes Dis. 4(2): 4363.

5. Ribotta MG (2001) Gene therapy strategies in neurodegenerative diseases. Histol Histopathol 16: 883-889.

6. Gray SJ, Woodard KT, Samulsk RJi (2010) Viral vectors and delivery strategies for CNS gene therapy. Ther Deliv 1(4): 517-534.

7. Nisbet RM, Polanco JC, Ittner LM, Götz J (2015) Tau aggregation and its interplay with amyloid- $\beta$. Acta Neuropathol 129(2): 207-220.

8. Bekris LM, Yu C, Bird TD, Tsuang DW (2010) Genetics of Alzheimer disease. J Geriatr Psychiatry Neurol 23(4): 213-227.

9. Barber RC (2012) The Genetics of Alzheimer's Disease. Scientifica. Article ID 246210.

10. Combarros 01, Alvarez-Arcaya A, Sánchez-Guerra M, Infante J, Berciano J (2002) Candidate gene association studies in sporadic alzheimer's disease. Dement Geriatr Cogn Disord 14(1): 41-54.

11. Hefti F (1986) Nerve growth factor promotes survival of septal cholinergic neurons after fimbrial transections. J Neurosci 6(8): 21552162.
12. Chen KS, Gage FH (1995) Somatic gene transfer of NGF to the aged brain: behavioural and morphological amelioration. J Neurosci 15(4): 28192825.

13. Martínez-Serrano A, Fischer W, Söderström S, Ebendal T, Björklund A (1996) Long-term functional recovery from ageinduced spatial memory impairements by nerve growth factor gene transfer to the rat basal forebrain. Proc Natl Acad Sci USA 93(13): 6355-6360.

14. Tuszynski MH, Yang JH, Barba D, U HS, Bakay RA, et al. (2015) Nerve Growth Factor Gene Therapy: Activation of Neuronal Responses in Alzheimer Disease. JAMA Neurol 72(10):1139-1147.

15. During MJ, Naegele JR, O’Malley KL, Geller AI (1994) Longterm behavioral recovery in Parkinsonian rats by an HSV vector expressing tyrosine hydroxylase. Science 266(5189): 1399-1403.

16. Kaplitt MG, Feigin A, Tang C, Fitzsimons HL, Mattis P et al. (2007) Safety and tolerability of gene therapy with an adeno-associated virus (AAV) borne GAD gene for Parkinson's disease: an open label, phase I trial. Lancet. 369(9579): 2097-2105.

17. Glorioso JC, Cohen JB, Carlisle DL, Munoz-Sanjuan I, Friedlander RM (2015) Moving toward a gene therapy for Huntington's disease. Gene Therapy 22(12): 931-933.

18. Stanek LM, Sardi SP, Mastis B Richards AR, Treleaven CM, Taksir T,et al (2014) Silencing mutant huntingtin by adeno-associated virus-mediated RNA interference ameliorates disease manifestations in the YAC128 mouse model of Huntington's disease. Hum Gene Ther 25(5): 461-474.

19. Paola Leone, David Shera, Scott W.J. McPhee, Francis JS, et al. (2012) Long-Term Follow-Up After Gene Therapy for Canavan Disease. Sci Transl Med. 4(165): 165ra163.

20. Eichle F, Duncan C, Musolino PL (2017) Hematopoietic Stem-Cell Gene Therapy for Cerebral Adrenoleukodystrophy. N Engl J Med 377: 16301638.

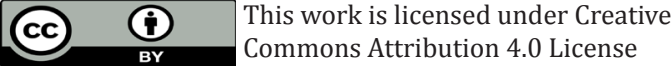

To Submit Your Article Click Here: $\quad$ Submit Article

DOI: $10.32474 /$ OJNBD.2018.01.000103

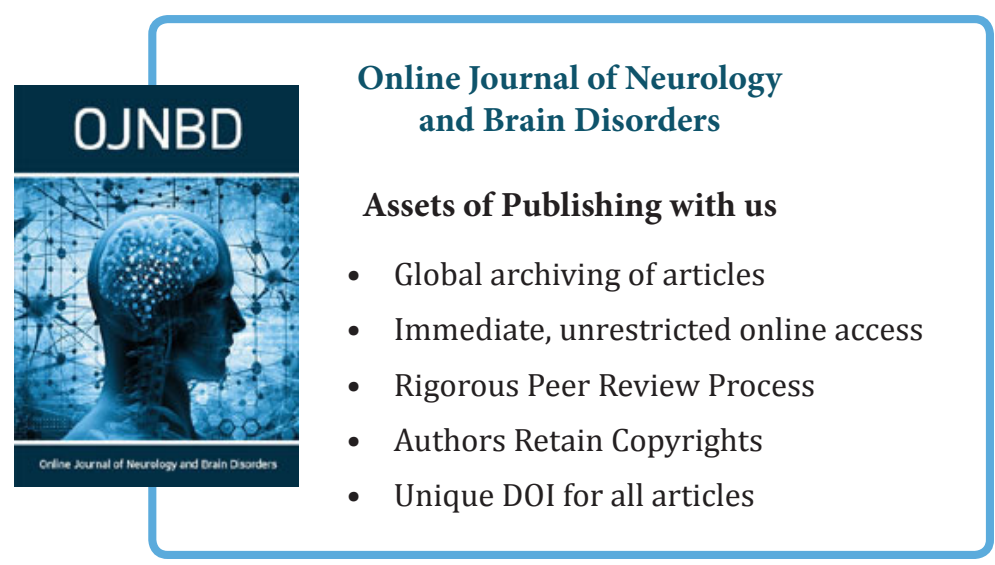

